\title{
ASPECTOS DA SITUAÇÃO DAS ESTATÍSTICAS OFICIAIS DA MORTALIDADE POR CAUSAS EXTERNAS NO MUNICÍPIO DE LONDRINA - PARANÁ1
}

\author{
SELMA MAFFEI DE ANDRADE 2
}

ANDRADE, S.M. de. Aspectos da situação das estatísticas oficiais da mortalidade por causas externas no Município
de Londrina - Paraná. Semina: Ci.Biol./Saúde, Londrina, v. 16, n. 2, p. 300-308, jun. 1995.

RESUMO: Analisou-se o comportamento da mortalidade por causas externas, no Município de Londrina-Paraná, segundo seus diferentes tipos: acidentes de trânsito, demais acidentes, suicídios, homicídios e lesס̃es em que se ignora se foram acidental ou intencionalmente infligidas, no período de 1979 a 1992. Evidenciou-se aumento estatisticamente significante para a mortalidade por acidentes de tránsito e por lesões em que se ignora se foram acidental ou intencionalmente infligidas (causa externa de tipo ignorado) e redução significativa para a mortalidade por homicídios. Comparação dos coeficientes em dois periodos diferentes, sendo um com base em dados oficiais (ano de 1992) e outro baseado em dados cujos atestados sofreram investigação para melhor definição das circunstâncias do evento que levou ao óbito (periodo de 10 de agosto de 1993 a 09 de agosto de 1994), evidenciou redução da mortalidade por lesões em que se ignora se foram acidental ou intencionalmente infligidas e aumento expressivo dos coeficientes de mortalidade por suicídios (81.8\%) e por homicídios (38.7\%). Os resultados sugerem que as estatísticas oficiais de mortalidade (periodo 1979 a 1992) podem estar subestimando causas especificas de mortalidade por suicídios e homicídios, principalmente. Entretanto, mesmo com as deficiências apresentadas, foi possível observar que os acidentes de trânsito, no contexto de Londrina, são um sério problema de saúde pública, predominando durante todo o periodo enquanto tipo de morte por causa extema e apresentando aumento significativo de seu coeficiente de mortalidade.

PALAVRAS-CHAVE: Estatísticas de mortalidade, causas externas, qualidade dos dados.

\section{1-INTRODUÇÃO}

Segundo a Organização Mundial de Saúde, causa externa de morte é aquela que sobrevem em conseqüência de um acidente ou outro tipo de violência, sendo, portanto, uma morte não natural (LAURENTI \& MELLO JORGE, 1983). De acordo com a legislação brasileira, todo óbito por causa externa deve ser atestado somente por médico legista, após necrópsia, ou por pessoas idôneas indicadas por autoridade judicial ou policial, nos locais que não disponham de postos do Instituto Médico-Legal (LAURENTI \& MELLO JORGE, 1983). Para fins estatísticos, essas causas de morte são classificadas de acordo com as rubricas da Classificação Suplementar de Causas Externas de Lesões e de Envenenamentos, do Manual de Classificação Estatística Internacional de Doenças, Lesões e Causas de Óbitos (CID-9) (ORGANIZAÇÃO MUNDIAL DA SAÚDE, 1985), subdividindo-se, basicamente, em acidentes de trânsito, demais acidentes (quedas, afogamentos, acidentes ambientais, entre outros), suicídios, homicídios, intervenções legais e resultantes de operação de guerra, e o grupo residual de lesões em que se ignora se acidental ou intencionalmente infligidas.

Em grande parte do mundo, as causas externas constituem-se em um expressivo problema de saúde pública, figurando entre as primeiras causas de mortalidade (BORBEAU, 1993). O impacto negativo dessas causas de morbidade e mortalidade se reflete na perda de inúmeras vidas, principalmente de jovens, no aumento da prevalência de incapacidades, no efeito de sobrecarga dos serviços de saúde, frequientemente já deficitários, e no conseqüente alto custo social e econômico para as populações (ORGANIZACIÓN PANAMERICANA DE LA SALUD, 1990).

Em que pese o fato de que as estatisticas de mortalidade representem apenas uma fatia minima do real problema que os acidentes e violências, de modo geral, representam para uma determinada comunidade, ainda assim são as mais largamente utilizadas em comparações entre diferentes locais ou em um mesmo local ao longo do tempo. Tal fato se deve, principalmente, à escassa disponibilidade de dados sobre a magnitude,

1 - Baseado em monografia apresentada à disciplina Epidemiologia das Doenças Năo Transmissiveis da Faculdade de Saúde Pública da USP, em novembro de 1994.

2- Departamento de Informações em Saúde/Autarquia de Saúde do Municipio de Londrina, Av. Duque de Caxias, 3499, Londrina, Pr, Brasil, CEP 86010-200.

- Departamento Materno Infantil e Saúde Comunitária/CCS - Universidade Estadual de Londrina, Caixa Postal 6001, Londrina, PR, Brasil, CEP 86051-990. 
o tipo e grau dos acidentes e violências e as sequielas não fatais resuntantes, entre Outros (ORGANIZACIÓN PANAMERICANA DE LA SALUD, 1994). Além disSO, outros aspectos positivos dos dados de monalidade são a obrigatoriedade de registro de cada monte a existência de séries lemporais, na maioria dos paises, possibilitando a anailise de tendencias.

No Brasil. segundo os unimos dados divulgados do Ministério da Saúde (MNISTERIO DA SAUDE, 1993), as causas extemas foram responsáveis por 102.252 mortes (12,5\% do total) em 1989, ocupando a segunda posição entre todos os grupos de causas, excluidas as mal definidas. Analisando os coeficientes de mortalidade por causas extemas nas capirais brasileiras em 1980 2 1988, alguns pesquisadores (ROUOUAYROL ê al., 1993) evidenciaram aumenio dessas laxas em 16 entre 26 capitais, especiamente em Porto Velno, 803 Vista, Maceió, Rio de Janeiro, São Paulo Cuiabå, cujos coeficientes se situavam, neste ühimo periodo, acima ou ao redor de 100 mortes por 100.000 habitantes. Ressalta-se o fato de que, no primeiro periodo (1980), o coeficiente máximo obsenado, por 100.000 habitantes, foi de 99,96 (Aracaju) e, ofio anos depois, este coeficiente máximo chegou 166,22 (Porto Velho). Segundo ainda os mesmos autores, houve um aumento da mortalidade proporcional por causas extemas no Brasil, que de $9,7 \%$ em 1979 passa para 11,53\% em 1988, deslocando-se da terceira para a segunda posiça entre as principais causas de morte.

Essas crescences laxas de monialiade por causas extemas não são, entretanto, una exclusividade da realidade brasineira. EshUdO da UNATED NATIONS (1982) revelou que, apesar de una reduça media de $12,2 \%$ para os coeficientes de rodas as causas de morte em 23 paises desenvolvidos, na faira etaria de 1 a 7 anos, o coeficiente de monialidade especifico por causas extemas sofreu um aumento de cerca de 6\%, no periodo entre 1955-59 e 1970-78, sendo este o maior incremento observado.

O compontamento que montalidade por causas extemas apresenta em diherentes locais, entretanto, difere quando analisadia nos seus divensos componemes. Enquanto que om alguns locais predominam os acidentes de transino, em ouros prevalecem os homiciolos ou suicidios ou ourros acidentes. Alguns paises, por exemplo, apresentam coeticientes de mortalioade especificos por suicidios maiores do que por acidentes de ir Japão, Noruega, Ássiria - Suịa. En outros ocorre 0 contrário, predominando as mortes por acidentes de

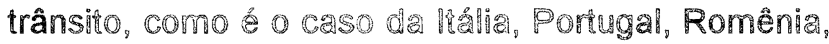
Espanha A Arstråa. Existem ouros paises, ainda, nos quais esses olois coeficientes sa praticamente equivalentes, cono no Chile, Remo Unido e Canadá (WORLDHEALTH STATISTICS ANNUAL, 199. Em outros locais, obsenv-Se unm mudancer a longo do

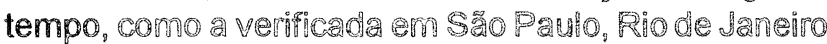
e Recife, Hocais nos quais os homicidios vêm

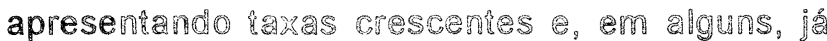
predominam enquan? causa specifica de morte na natural (MELLO JORGE, 1982; MENEZES FILHO et al., 1990; MUNAYO \& SOUZA, 1993; SOUZA, 1993). EsSa hererogeneidade deve-se aos diferentes contextos sociais, policicos e economicos vivenciados pelas diferenies comunioades e o connecimento aprofundado de como, em que quantidade e porque ocorrem tais fenômenos deve ser buscado para que estratégias que visem ì reduça de lais eventos possam ser postas em prática.

A análise dos coeficientes especificos de mortalidade por causas externas segundo seus diferentes subgrupos, entretanto, pode ser dificuliada em razão da qualidade dos dados disponiveis. Inumeros estudos têm demonstrado que, em geral, as causas de morte informadas no atestado de óbito nem sempre correspondem realidade (LAURENTI \& MELLO JORGE; 1983: LAURENTI, 1993; MELLO JORGE; 1988; MELLO MORGE, 1990; MOUSSA, 1990; PUFFER \& GRIFFITH, 1967: SCHNITMAN, 1990). NO CASO especifico dis mortes por causas extemas, como ressalta RELLO JORGE (1988; 1990), devido a diversos fatores, grande parte dos atestados de óbito descrevem aponas as causas conseqüenciais (trammatismo, hemorragia ou lesões) encontradas na necrópsia, sem qualquer peferencia às "circunstâncias do acionene ou

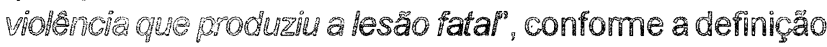
da Onganizaça Mundial de Saúde (1985) sobre causa băsica de mone năo natural. Isto faz com que grande parte dos óbiros por causa extema seja classificada sob as rubricas E980. E989 da CID-9 (lesões em que Se ignora se acidental ou intencionalmente infligidas), que compồn nma categoria residual de causa de morte na๊o natural a que, portanto, pouco informa no sentido de que medidas preventivas deveriam ser adotadas para evirar tais nontes.

Estudo envolvendo o Brasil e mais nove paises, na década 0 00, evidenciou, por exemplo, que diversas causas de monte nromadas no atestado oleverian ser 3:eradis apos uma investigaçă mais detahada, ąu envolvia entrevistas com familiares, consultas a pronumios hospinalares e outros locais. No caso especifico de mortes por causas externas, de 4.248 obion de pessoas entre 15 e 74 anos, 202 foram reclassificados em outros grupos de causa. Por ourto lado, outras 303 mortes originalmente chassincadas como ole cansas naturais foram inconporadas a grepo de causa expern. Aem disso, 1.280 causas de morte videnta Poram reclassificadas dentro do próprio grupo. As causas mais subestimadas, neste grupo, foram os suicidios, oue passaram de 536 originamente infomados para 98: 0s homicidios, que de 482 passaram para 843; c os acidentes de trânsito, de 721 para 1.243, com

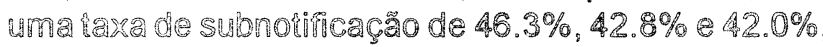
TESpechivamene (PUFFER \& GRIFFITH, 1967).

Em un Urabaho mais recente, MELLO IORGE (1990), uribmando metodologia semehante, comparou as osthínicas oficiais de morte por causas extemas em menores de 15 anos com as que defimiu após minuciosa investigaça, chegando à conclusão que, em Så Pald, "ao menos na populaça oxaminade de 
menores de 15 anos -, as estatísticas oficiais deixam a desejar." , com uma concordância de apenas $24,05 \%$ na categoria da causa básica de morte e de $67,13 \%$ para agrupamento.

Para contornar essa dificuldade, trabalho descritivo sobre a violência na cidade do Rio de Janeiro incluiu, no cálculo dos homicídios propriamente ditos, as agressões por armas de fogo, as quais se ignorava se acidental ou intencionalmente infligidas. Os autores acreditavam que, com base em informações de delegacias de polícia, este procedimento aproximava mais os dados da realidade (MINAYO \& SOUZA, 1993). Com a correção efetuada, o coeficiente de mortalidade por homicídios passou de pouco mais de 5 para cerca de 35 por 100.000 habitantes, no ano de 1984, por exemplo, evidenciando o quanto os registros oficiais podem subdimensionar o problema real dessa causa específica de morte não natural.

Este trabalho tem como finalidade analisar o comportamento das causas externas de morte no município de Londrina, estado do Paraná, verificando a evolução dos coeficientes dos diferentes tipos de morte não natural, destacando a situação das causas externas de morte por lesões em que se ignora se foram acidental ou intencionalmente infligidas, no período de 1979 a 1992. Além disso, pretende levantar hipóteses quanto às possíveis causas específicas de morte por causa externa subestimadas nas estatisticas oficiais de mortalidade, comparando os coeficientes de cada tipo de morte por causa externa em dois periodos: o primeiro, de 01 de janeiro a 31 de dezembro de 1992 (dados oficiais, sem qualquer investigação adicional) e o segundo, de 10 de agosto de 1993 a 09 de agosto de 1994 (dados processados após investigação, embora não incluindo os ocorridos fora do Município de Londrina). Com a comparação dos coeficientes desses dois periodos, objetiva-se verificar, com a possivel redução das mortes por causas em que se ignora se acidental ou intencionalmente infligidas (causa externa de tipo ignorado), quais os tipos especifícos de causa de morte que sofreriam aumento em seus coeficientes.

\section{2-MATERIAL E MÉTODO}

\section{1- Local de estudo}

Londrina é uma cidade com história recente, fundada em 1930 e elevada à categoria de municipio em 1934, cuja origem esteve associada à expansão da fronteira agrícola do café, com a intensa imigração, principalmente de paulistas e mineiros. Tornou-se, em pouco tempo, uma das mais importantes cidades do norte do estado do Paraná, sendo, atualmente, a segunda e terceira mais populosa cidade do estado e da região Sul, respectivamente. Com a crise vivenciada pela cultura cafeeira, a partir da década de 60, a implantação de uma política agrária voltada para a produção de soja e trigo, com conseqüente mecanização da agricultura, e a crescente industrialização, grandes contingentes de trabalhadores nurais migraram para as cidades ou outras regiões. Com isso, o processo de urbanização em
Londrina foi bastante rápido. Enquanto aproximadamente $40 \%$ da população vivia na cidade, em 1970 , essa proporção passa para $94 \%$ em 1991. Conseqüência desse processo, como referido por LAPREGA (1989), foi o "aumento do cinturáo de pobreza formado por massas de desempregados, empregados do setor industrial ou bóias-frias", quadro tipico de diversas áreas metropolitanas brasileiras. No último recenseamento geral, Londrina contava com 389.959 habitantes, com um crescimento populacional de 2,36\% 20 ano, de 1980 a 1991 (FUNDAÇÃO IBGE, 1991).

Com relação ao perfil de montalidade por causas, com exceção de trabalho feito por LAPREGA (1989), as únicas informações disponiveis, para o Município, se referem às listas de mortalidade do Subsistema de Informações de Mortalidade, implantado pelo Ministério da Saúde, a pariir do ano de 1979. Ainda que o já referido estudo de LAPREGA tenha objetivado estudar a invasão de óbitos no Município, de 1936 a 1982, este oferece contribuição para o estudo da evolução do perfil da mortalidade proporcional por causas desde a década de 30 , em Londrina.

Na década de 30 , as causas extemas de morte foram responsáveis por $7,7 \%$ da mortalidade de residentes em Londrina, sendo menos freqüente, apenas, que as afeç̧ões mal definidas $(32,7 \%)$, as doenças infecciosas e parasitárias $(29,0 \%)$ e as doenças do aparelho respiratório $(10,4 \%)$. Naquela época, os acidentes de trânsito representavam apenas $2,2 \%$ das causas externas de morte e chama a atenção a alta proporça de mortes intencionais - homicidios e suicidios, representando $20,0 \%$ e $8,9 \%$, respectivamente, do total de mortes por causas externas. $\mathrm{Na}$ década de 60 , as causas extemas representaram $5,4 \%$ do total de mortes, predominando os demais acidentes e os acidentes de irânsito, que responderam por cerca de $51 \%$ e $20,2 \%$ das mortes por causas externas, respectivamente. Na década de 70, a mortalidade proporcional por causas extemas passa para $8,7 \%$ e os demais acidentes e os acidentes de Irânsito continuam predominando, representando cerca de $41,0 \%$ e $38,6 \%$ dos óbitos deste grupo de causa de morte, respectivamente (LAPREGA, 1989).

Em relação às mortes por lesöes em que se ignora se acidental ou intencionalmente infligidas, o mesmo estudo revelou uma razoável proporç̃o em relação ao total de causas extemas, nas décadas de 30, 40,50 e 60: $11,1 \%, 12 \%, 10,5 \%$ e $12,8 \%$, respecivamente. Na década de 70 , a proporção desta causa apresentou significativa redução, representando apenas $1,4 \%$ das mortes por causas extemas, indicando uma melhoria da informação desse grupo de causa de morte. nessa década específica.

\subsection{Material metodologia}

Para a primeira pante deste trabalho, o material constituiu-se dos dados de mortalidade consolidados pela Secretaria de Estado da Saúde do Paraná, através das listas de número de óbitos por 1000 causas segundo sexo e idade, de 1979 1992, para residentes em 
Londrina. Para os cálculos dos coeficientes foi utilizada a população anual estimada pela Secretaria de Planejamento do Municipio de Londrina, cujas estimativas basearam-se nos censos demográficos de 1980 e 1991.

Com o objetivo de faciliự a anălise da tendência dos coeficientes, que apresentam flutuações aleatórias devido a pequenos numeros, o periodo de 14 anos foi agrupado em dois de 7 anos cada: de 1979 a 1985 e de 1986 a 1992. Para a comparação das diferenças dos coeficientes específicos entre os dois periodos foi utilizado o teste "z", bicaudal, num nivel de significância de $5 \%(\alpha=0.05)$, com os respectivos intervalos de confiança de $95 \%$ para cada diferença observada (DALY et al., 1991).

As causas exiemas de morte definidas nesse trabaiho baseiam-se na Classificação Internacional de Doenças (ORGANIZACÃO MUMDIAL DA SAUDE, 1985), nona revisão, que se encontram sob as rubricas E800 a E999 da Classificaço Suplementar de Causas Extemas de Lesões e de Envenenamentos. A agregação quanto aos tipos de acidentes ou violências foi a mesma utilizada em trabalho de MELLO JORGE (1982), isto é:

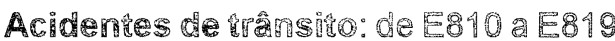

Demaำ a E849

Suicidios: de E950 a 5959

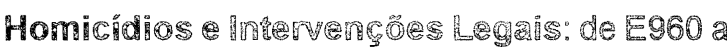
E978, neste trabalno denominadas apenas como "homicidios"

Lesões an que \$2 ígror ou intencionalmente intligidas: de E980 a E989, neste trabalho denominadas como "causa ignorada"

Para a segunda parte deste trabalho compararam-se os coeficientes especificos de mortalidade por causa extema segundo o agrupamento acima em dois períodos distintos, porém próximos no tempo, de 12 meses cada. O primeiro periodo compreendeu a ano de 1992 (de 01 de janeiro a 31 de dezembrol, cujos coeficientes foram calculados utilizando os dados oficiais da Secretaria de Estado da Saúde, confome já descrito acima. O segundo periodo compreendeu as montes por causas extemas ocorridas de 10 de agosto de 1993 a 09 de agosto de 1994, período este no qual o municipio comecou a trabahar os dados de mortalidade e ostem disponiveis, portanto, em banco de dados infomatizado, autravés do Núcleo de Infomação em Mortalidade (NM). Para esie segundo periodo, os dados foram processados eletronicamente através do programa Subsistema de Infomaçőes sobre Mortalidade (SIM) do Ministério da Saúde, senco que, para este trabalho, foram lidos e tabulados ấravés do programa Epilnfo (DEAN et al., 1990).

A diferenga essencial entre o primeiro e segundo periodo é que, mo primeiro (1932), as causas de morte eram codificadas e processadas da forma como informadas na Declaraça de Obiro (0.0.), ou seja, sem qualquer intenenço de mehoria dos dedos. No segundo período (1993/94), com a municipalização da coleta processamento dos dados de mortalidade de residentes, todos os óbitos por causa externa, entre outras, foram investigados através de consultas ao Institu? MédicoLegal, Plantão de Acidentes, familiares, hospirais elon em noticias veiculadas na imprensa escrita loca! permitindo, na grande maioria das vezes, definir mais precisamente as circunstâncias do acidente ou violência que conduziu à morte. Acredita-se, portanto, que os dados referentes a este segundo periodo sejam de melhor qualidade do que os do ano de 1992.

Comparando os coeficientes específicos para cald tipo de morte por causa externa, esperou-se verifica quais os coeficientes que apresentaram, no segundo periodo, maior incremento proporcional e que, pontanto, poderiam estar subestimados nos anos anteriores.

Eimportante ressaltar, entretanto, que enquanto os dados de mortalidade do primeiro periodo (1992) se referem aos óbitos de residentes ocorridos ou na: no Município de Londrina, os do último período 110 de agosio de 1993 a 09 de agosto de 1994) se referem apenas aos de residentes ocorridos no próprio Município. No entanio, sendo Londrina um pólo de atração para assistência médico-hospitalar, pressupõe-se que a evasão de óbilos seja pequena, restringindo-se principalmente aos acidentes de trânsito ocorridos fora dos limites geográficos do Município. A título de comparaça, no ano de 1992 foram registrados um total (ocorridos ou não no Municipio) de 2.181 óbitos de residentes em Londrina. No periodo de 10 de agosto de 1993 a 09 de agosto de 1994, apenas para os óbitos de residentes ocorridos no próprio Município, este total roi bastante próximo, ou seja, 2.110 óbitos. Ainda assim, os coeficientes considerados neste último periodo especifico (1993/94) devem ser considerados cono "minimos", com possibilidades de aumento, lembrando que cerca de 4 mortes a mais por determinada causa aumentaria o coeficiente dessa causa em iomo de $1 /$ 100.000 habitantes.

\section{RESULTADOS E DISCUSSÃO}

O Gráfico 1 apresenta a evolução dos coeficientes de mortalidade por causas externas e seus subgrupos em Londrina, nos últimos 14 anos, cujas estatisticas foram consolidadas pela Secretaria de Estado ola Saúde do Paraná e são, portanto, oficiais. Pode-se observar que as taxas de mortalidade por causas extemas, $n 0$ periodo, pemaneceram praticamente estáveis. Por oufro lado, as laxas por diversos tipos de causas extemas apresentaram diferenças importantes. Obsenvase que a mortalidade por acidentes de trânsito fol a lue predominou em todo o periodo, com tendencia de crescimento a partir de 1982. Os demais subogrupos apresentam tendência menos caracteristica, con flutuações positivas ou negativas ao longo dos anos. A única exceção é o coeficiente de mortalidade por "causa ignorada", que de 1979 a 1986 vinha apresentando declínio e que, em 1987, apresenta uma elevaço Sindo permanecendo elevado nos anos subsequentes. 


\section{GRÁFICO 1 - COEFICIENTES DE MORTALIDADE (POR 100.000 HABITANTES) POR CAUSAS EXTERNAS E SEUS SUBGRUPOS DE RESIDENTES EM LONDRINA-PR, DE 1979 A 1992}

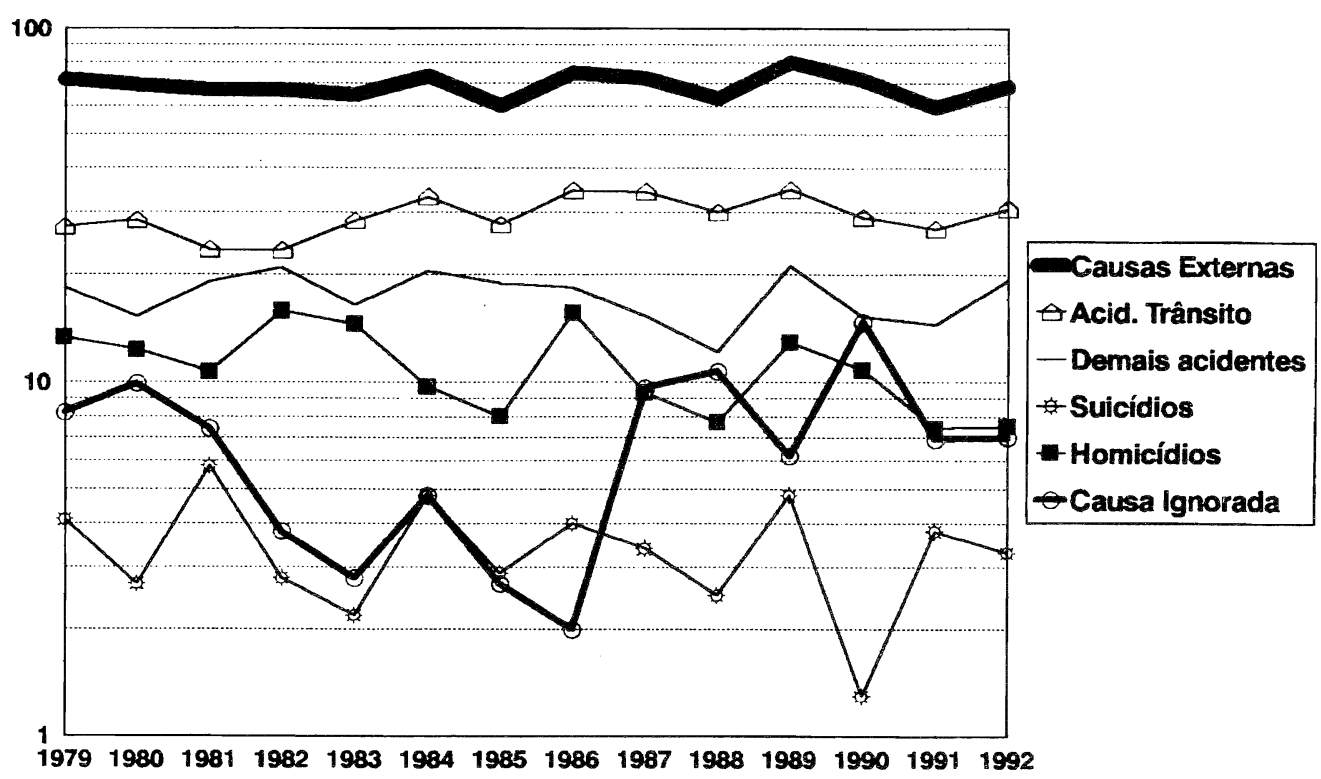

A Tabela 1 apresenta os dados já agrupados em dois períodos: o primeiro referente aos anos de 1979 a 1985 e, o segundo, de 1986 a 1992 . Como pode-se observar, não houve diferença estatisticamente significativa para os coeficientes de mortalidade por demais acidentes, suicídios e 0 total de causas externas entre os dois periodos observados. O mesmo não ocorreu com a taxa de homicídios, que apresentou redução estatisticamente significante $(p<0.05)$, com os acidentes de trânsito, que apresentaram aumento $(p<0.02)$ e, principalmente, com as mortes por causa ignorada, cujo aumento de coeficiente foi 0 mais expressivo $(p<0.01)$. Ressalta-se que, em relação ao período de 1979-1985, o aumento relativo deste último coeficiente foi o mais expressivo $(47,75 \%)$, apesar de um aumento de coeficiente de apenas 2,65 por 100.000 habitantes (Gráfico 2).

TABELA 1 - COEFICIENTES DE MORTALIDADE (POR 100.000 HABITANTES) POR CAUSAS EXTERNAS E SEUS SUBGRUPOS, EM 1979-85 E 1986-92, DIFERENÇAS DOS COEFICIENTES NOS DOIS PERIODOS, ZOBSERVADO E INTERVALO DE CONFIANÇA DE 95\% PARAAS DIFERENÇAS OBSERVADAS, DE RESIDENTES EM LONDRINA-PR.

\begin{tabular}{|c|c|c|c|c|c|}
\hline Causa de morte & $\begin{array}{l}\text { Coeficientes } \\
1979-85 \\
\text { (A) }\end{array}$ & $\begin{array}{l}1986-92 \\
\text { (B) }\end{array}$ & $\begin{array}{l}\text { Diferença dos Coeficientes } \\
(B-A)\end{array}$ & $\begin{array}{l}Z \text { observado } \\
\text { (bicaudal) }\end{array}$ & $\begin{array}{l}\text { Intervalo de Confiança } 95 \% \\
\qquad /(B-A)\end{array}$ \\
\hline Ac. Trânsito & 27,51 & 31,40 & $+3,89$ & $+2,48^{*}$ & $+0,83 a+6,95$ \\
\hline Demais acidentes & 18,52 & 16,56 & $-1,96$ & $-1,63$ & $\cdot 4,33 a+0,41$ \\
\hline Suicidios & 3,61 & 3,29 & $-0,32$ & $-0,60$ & $-1,37 a+0,73$ \\
\hline Homicídios & 11,97 & 10,08 & $-1,89$ & $-1,99^{* *}$ & $-3,77 a-0,01$ \\
\hline Causa ignorada & 5,55 & 8,20 & $+2,65$ & $+3,49^{* * *}$ & $+1,18 a+4,12$ \\
\hline $\begin{array}{l}\text { Causas externas } \\
\text { (Total) }\end{array}$ & 67,18 & 69,55 & $+2,37$ & $+0,99$ & $-2,31 a+7,05$ \\
\hline${ }^{*} p<0.02$ & $<0.05$ & $<<0.01$ & & & \\
\hline
\end{tabular}



1985, RESIDENTES EM LONDRINA-PR

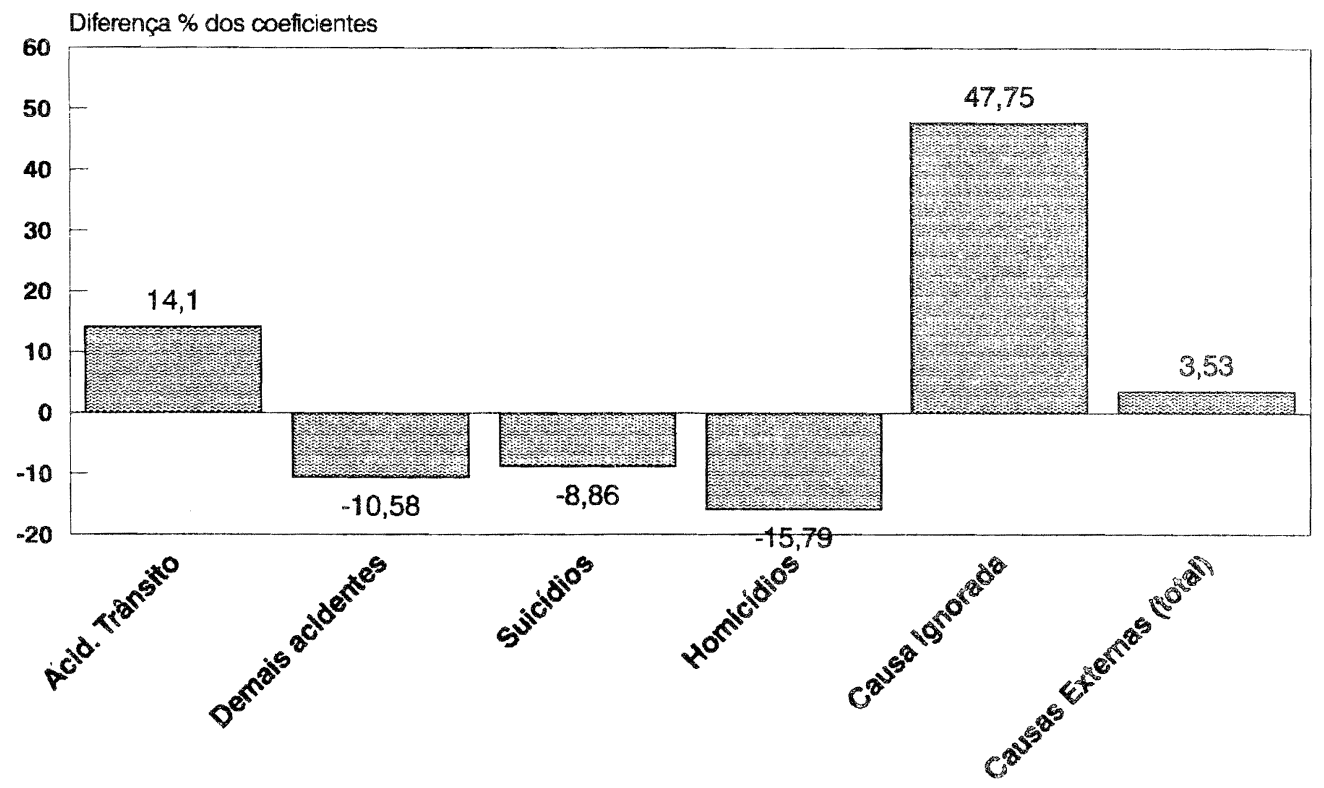

Este aumento significativo de mortes por causa ignorada (lesões em que se ignora se foram acidental ou intencionalmente infligidas) reflete uma piora do sistema oficial de registro de mortes por causas externas em Londrina, no período analisado. Este fato prejudica a elaboração de um diagnóstico completo e correto da situação particular da mortalidade por causas externas no Município e, conseqüentemente, a possibilidade de adoção de estratégias apropriadas para a redução dos agravos mais importantes. É importante ressaltar, todavia, que este deterioramento da qualidade da informação não é exclusividade de Londrina (HÍJAR MEDINA et al., 1986; MELLO JORGE, 1988; MELLO JORGE, 1990; SOUZA, 1993; SZWARCWALD \& CASTILHO, 1986; YUNES, 1993). Mesmo em países desenvolvidos, como Portugal, Suécia, Inglaterra e Gales, observou-se uma proporção relativamente alta de mortes violentas em que se ignorava a intencionalidade, com valores aproximados de $16 \%, 11 \%$ e $12 \%$, respectivamente, em relação ao total de mortes por causa externa (BOURBEAU, 1993). O mesmo autor revela, por outro lado, que a situação é extremamente mais grave em países em desenvolvimento, como no Chile e Martinica, cujas proporções de morte por causa externa ignorada se situam ao redor de $50 \%$. No Brasil, a situação é bastante heterogênea, com valores variando entre próximos de zero a cerca de $50 \%$ (MELLO JORGE, 1990). A observação dessas taxas é importante, no entanto, pois verifica-se que existem possibilidades de minimização da proporção dessas causas externas de tipo ignorado.

Um coeficiente elevado de mortalidade por causa ignorada pode estar, em diferentes graus, subestimando a magnitude de outras causas específicas de mortalidade por causa externa. A questão que se coloca, no caso de Londrina, é até que ponto as diferenças entre os coeficientes dos dois períodos, isto é, a não diferença significativa observada para o grupo dos demais acidentes e suicídios, ou a redução significativa apresentada pelos homicídios, são reais ou simplesmente resultados desse aumento de causas ignoradas.
Alguns trabalhos têm demonstrado a existência de associação negativa entre o grupo de causas externas ignoradas (E980-E989) e o grupo de homicídios (E960E969), ou seja, à medida que um coeficiente aumenta o outro diminui. Esta associação foi extremamente significativa $(r=-0,95)$ em estudo realizado no México, por exemplo (HIJARMEDINA et al., 1986). A metodologia utilizada, entretanto, não permite assegurar que a maior proporção das causas ignoradas seja, na realidade, homicídios. Como bem lembrado pela Organização Panamericana de Saúde (1994), em muitos países, devido a preconceitos religiosos ou culturais, muitas das mortes por suicídios podem não ser classificadas como tais, sendo uma das possibilidades a classificação sob as rubricas de lesões em que se ignora se acidental ou intencionalmente infligidas.

A metodologia ideal para responder à questão acima colocada, ou seja, que causas bem definidas estäo "ocultas" no grupo de causas ignoradas, seria a adotada. por diversos autores (ALLEBECK et al., 1991; MELLO JORGE, 1988, 1990; MOYER et al., 1989; PUFFER \& GRIFFITH, 1967). Por dificuldades práticas, entretanto, neste trabalho optou-se por comparar os coeficientes de cada tipo de morte por causa externa em dois períodos distintos, conforme descrito anteriormente e analisado a seguir.

Analisando os dados da Tabela 2, pode-se verificar que, apesar de os coeficientes do segundo período serem mínimos, isto é, passíveis ainda de aumento, houve um aumento considerável para os grupos dos suicídios $(81,8 \%$ ) e dos homicídios ( $38,7 \%)$, em relação ao último coeficiente apresentado. Considerando que a situação conjuntural/estrutural, em Londrina, não sofreu mudanças radicais entre um e outro período, pode-se levantar a hipótese de que esses aumentos observados se deveram, principalmente, à melhoria dos dados no segundo período e que, portanto, os coeficientes anteriores de mortalidade por causa ignorada estavam, na realidade, camuflando mortes por suicídios e homicídios, principalmente. 


\section{TABELA 2 - COEFICIENTES DE MORTALIDADE (POR 100.000 HABITANTES) POR CAUSAS EXTERNAS E SEUS SUBGRUPOS NO ANO DE 1992 E NO PERIODO DE 10 DE AGOSTO DE 1993 A 09 DE AGOSTO DE 1994 E DIFERENÇA PERCENTUAL DOS COEFICIENTES DO ÚLTIMO PERÍODO EM RELAÇÃO AO PRIMEIRO, LONDRINA-PR.}

Causa de morte 1992 Coeficientes

Acid. de Trânsito

Demais acidentes

Suicídios

Homicídios

Causa ignorada

Causas externas (total)
(A)

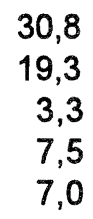

67,9
$1993 / 94^{\star \star}$

(B)

$\begin{array}{rr}25,1 & -18,5 \\ 16,6 & -14,0 \\ 6,0 & +81,8 \\ 10,4 & +38,7 \\ 1,0 & -85,7 \\ 59,1 & -13,0\end{array}$

* Coeficientes oficiais (residentes em Londrina, óbitos ocorridos ou não no Município).

** Período de 10.08.93 a 09.08.94, coeficientes mínimos (residentes em Londrina, óbitos ocorridos no próprio Município).

Chama a atenção, ainda, o fato de quase a totalidade da redução do coeficiente por causa ignorada (6,0 por 100.000 habitantes) ter sido distribuída entre os homicídios $(2,9 / 100.000)$ e os suicídios $(2,7 / 100.000)$, totalizando 5,7 por 100.000 habitantes.

Estes resultados indicam, em resumo, que qualquer conclusão a respeito do comportamento da mortalidade por homicídios e suicídios, em Londrina, que se baseie exclusivamente nas estatísticas oficiais disponíveis (de 1979 a 1992), pode ser equivocada. Em outras palavras, há indicações de que a não diferença ou a redução significativa observadas para os coeficientes de mortalidade por suicídios e homicídios, respectivamente, são, na realidade, meros artefatos da queda de qualidade da informação. Nesse contexto, a municipalização da coleta e processamento da informação de mortalidade foi uma estratégia importante para a melhor definição das causas básicas das mortes por causas externas de residentes em Londrina, com uma redução expressiva do coeficiente de mortalidade por causas ignoradas (de 7,0 para 1,0 por 100.000 habitantes).

Ainda que as estatísticas oficiais apresentem as deficiências apontadas acima, os dados evidenciam que os acidentes de trânsito são um sério problema de saúde pública em Londrina, a exigir maiores estudos e ações multidisciplinares e intersetoriais que visem à sua redução. Além de predominarem, enquanto tipo específico de morte por causa extema, em todo o periodo, ainda apresentaram aumento significativo $(p<0.02)$ do coeficiente de mortalidade no período 1986-1992 em relação ao período anterior (1979-1985). Além disso, o coeficiente mínimo apresentado de 10 de agosto de 1993 a 09 de agosto de 1994 (25,1 por 100.000 habitantes), que engloba apenas os óbitos de residentes ocorridos no próprio Município, pode ser considerado alto quando comparado a outros locais (BOURBEAU, 1993; SOUZA, 1993; WORLD HEALTH STATISTICS ANNUAL, 1994; YUNES, 1993). Este coeficiente, na realidade, se traduz na perda da vida de uma pessoa, geralmente jovem, a cada três dias e meio, para um Municipio com pouco mais de 400.000 habitantes.

\section{4-CONCLUSÕES}

Os resultados apresentados neste trabalho permitiram concluir, em relação à mortalidade por causas externas, em Londrina:

4.1. Houve uma piora significativa do sistema oficial de estatísticas de causa de morte no que se refere às causas externas, no período de 1979-1992;

4.2. Apesar dessa piora do sistema de informação sobre mortalidade por causa externa, evidenciou-se aumento significativo do risco de morte por acidentes de trânsito, no período acima citado, situação esta que está a exigir ações que visem à minimização deste problema;

4.3. O aumento observado do coeficiente de mortalidade por lesões em que se ignora se foram acidental ou intencionalmente infligidas não permite concluir que a redução significativa do risco de morte por homicídios ou a diferença não significativa do risco de morte por suicídios, observados neste trabalho, sejam reais ou simplesmente resultantes da piora da qualidade dos dados referentes às causas externas de morte;

4.4. A municipalização da coleta e processamento dos dados de mortalidade permitiu uma redução expressiva do coeficiente de mortalidade por causas externas as quais se ignorava se acidentais ou intencionais (de 7,0 para 1,0 por 100.000 habitantes);

4.5. Com a redução do coeficiente de mortalidade por causas externas ignoradas, observouse um aumento relativo dos coeficientes de mortalidade por suicídios $(81.8 \%)$ e homicídios $(38,7 \%)$ em relação ao último dado oficial divulgado (1992), possibilitando aventar a hipótese de que estas duas causas especificas de morte estavam sendo subestimadas nas estatísticas oficiais dos anos anteriores. 
ANDRADE, S.M. de. Aspects of the situation regarding official mortality statistics from extemal causes in the municipality of Londrina, Paraná State, Brazil. Semina:Ci.Biol./Saúde, Londrina, v. 16, n. 2, p. 300-308, Jun. 1995.

ABSTRACT: Mortality from external causes has been analyzed in Londrina, Parand State, Brazil, accoroling lo its different types: traffic accidents, other accidents, suicides, homicides and deaths from undetemined mientionality (unknown causes), from 1979 to 1992. A statistically significant increase has been demonstrated for montanity from traffic accidents and from undetermined intentionality. On the other hand, homicide rates have shown a statistically significant reduction. Comparison between rates of two periods, one of them based on offcial date 11992$)$ and the other based on data whose death certificates had been subjected to deeper imvestigation (August 10, 1993 to August $09,1994)$ to better identify the circunstances of the event which has led to death, has shown arn Expressive reduction

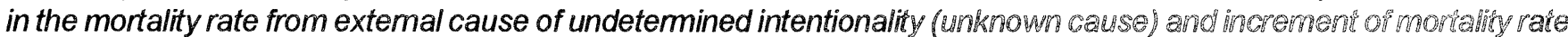
from suicide (81.8\%) and homicide (38.7\%). Results suggest that official montality data (from 1979101992$) \mathrm{m}$ may be underestimating specific causes of non-natural death such as suicide and homicide, mainfy. Notwithstanding these official data deficiencies, it has been possible to detect that traffic accidenis are a major healih problem in londrina, prevailing during the entire period as specific type of death from external cause and presening increasing rates.

KEY-WORDS: Mortality statistics, external causes of death, quality of da?'?

\section{REFERÊNCIAS BIBLIOGRÁFICAS}

ALLEBECK, $\mathrm{P}$, et al. Causes of death in a cohort of 50,465 young men: validity of recorded suicide as underlying cause of death. Scand. J. Soc. Med., v. 19, n. 4, p. 242-7, 1991.

BOURBEAU, R. Analyse comparative de la mortalité violente dans le pays développés et dans quelques pays en développement durant la période 1985-1989. World Health Stat. Q., v. 46, n. 1, p. 4-33, 1993.

DALY, L.E. et al. Interpretation and uses of medical statistics. 4. ed. Oxford: Blackwell Scientific Publications, 1991.

DEAN, A.G. et al. Epi Info, version 5: a word processing, database, and statistics program for epidemiology on microcomputers. Atlanta: Centers for Disease Control, 1990.

FUNDAÇÃO IBGE. Censo demográfico: sinopse preliminar: Paraná. Rio de Janeiro: Fundação IBGE, 1991, v。 6, n. 4 (10o. Recenseamento Geral do Brasil).

HÍJAR MEDINA, M.C. et al. Mortalidad por accidentes, violenciais $y$ envenenamientos en el Distrito Federal de 1970 a 1982. Salud Pública de Méx., v. 28, n. 4, p. 413-37, 1986.

LAPREGA, M.R. Evoluçåo da mortalidade no municipio de Londrina, Paraná de 1936 a 1982: contribuição dos óbitos invasores. Ribeirão Preto, 1989. Dissertação (Mestrado) - Faculdade de Medicina da USP.

LAURENTI, R.; MELLO JORGE, M.H.P. de. O atestado de óbito. São Paulo: Centro Brasileiro de Classificação de Doenças, 1983. (Série Divulgação n。 1).

LAURENTI, $R$. Maternal mortality in Latin American urban areas: the case of São Paulo, Brasil. Bull. Pan. Am. Health Organ., v. 27, n. 3, p. 205-14, 1993.

MELLO JORGE, M.H.P. de. Mortalidade por causas violentas no Município de Säo Paulo, Brasil. IV - A situação em 1980. Rev. Saúde Pública, v. 16, n. 1, p. 19-41, 1982.

MELLO JORGE, M.H.P. de. Investigação sobre a mortalidado por acidentes e violéncias na infáncia. São Paulo, 1988. Tese (Livre-Docência) - Faculdade de Saúde Pública da USP.

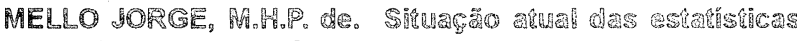

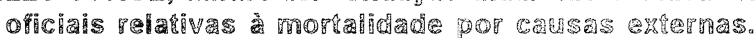

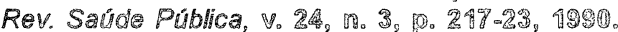

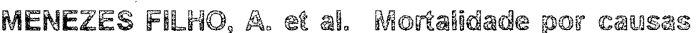

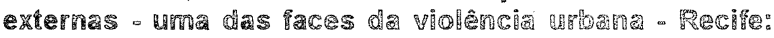
1977-1985. Un: CONGRESSO BRASHLEURO DE EPIDEMIOLOGIA, 10, Campinas, 1980. Programa. Rio de Janeiro: ABRASCO, 1990. TR. 144.

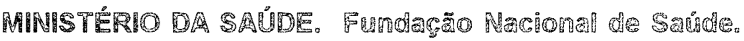
Centro Nacional de Epiodemiologia. Estatisticas de

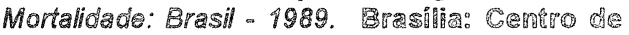

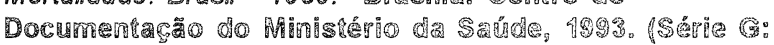
Estatistica m m

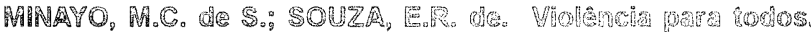

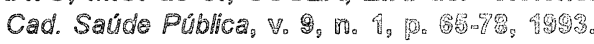

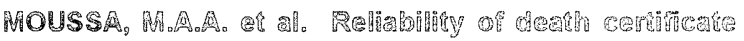

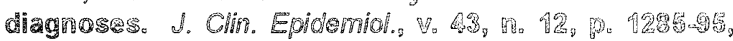
1990.

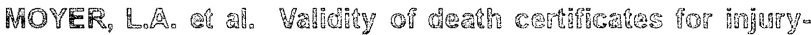

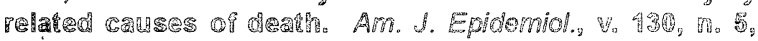
P. 102奥32, 1982.

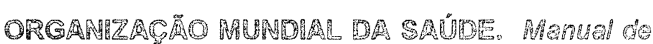

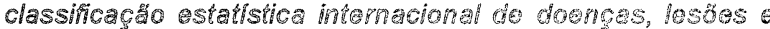

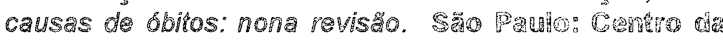

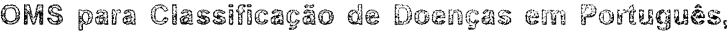
1985. V. 1.

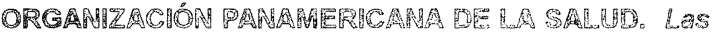
condiciones de salud en las Americas. Mhashingegen:

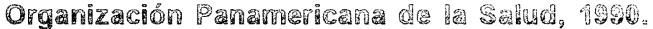

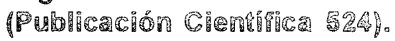

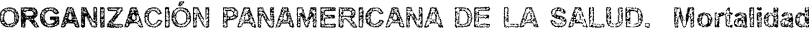

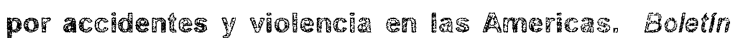

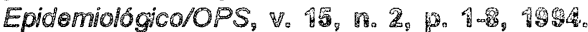

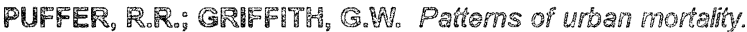

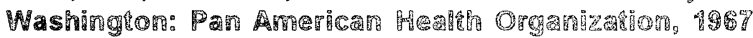

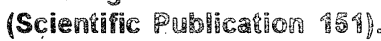

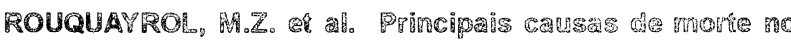

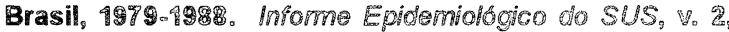


n. 5, p. 28-37, 1993.

SCHNITMAN, A. Análise da fidedignidade da declaração da causa básica de morte por câncer em Salvador, Brasil. Rev. Saúde Pública, v. 24, n. 6, p. 490-6, 1990.

SOUZA, E.R. de. Violência velada e revelada: estudo epidemiológico da mortalidade por causas externas em Duque de Caxias, Rio de Janeiro. Cad. Saúde Pública, v. 9, n. 1, p. 48-64, 1993.

SZWARCWALD, C.L. \& CASTILHO, E.A. de. Mortalidade por causas externas no estado do Rio de Janeiro no período de 1976 a 1980 . Cad. Saúde Pública, v. 2, n. 1 , p. $19-41,1986$.

UNITED NATIONS. Levels and trends of mortality since 1950: a joint study by the United Nations and the World Health Organization. New York, 1982.

WORLD HEALTH STATISTICS ANNUAL, 1993. Genève: World Health Organization, 1994.

YUNES, J. Mortalidad por causas violentas en la región de las Americas. Bol. Oficina Sanit. Panam., \%. 114, p. 302-16, 1993. 